Ann. Biol. anim. Bioch. Biophys., I967, 7 (2), r45-155.

\title{
CROISSANCE DES TISSUS DU RAT ET RÉDUCTION DE L'APPORT ENERGÉTIQUE DE LA RATION; INFLUENCE SUR LA TENEUR EN ACIDES NUCLÉIQUES
}

\author{
G. DURAND, G. FAUCONNEAU et Éliane PENOT \\ Station centrale de Nutrition, \\ Centre national de Recherches zootechniques, 78 - Jouy-en-Josas \\ Institut national de la Recherche agronomique
}

SOMMAIRE

La croissance du foie, de la musculature des membres postérieurs et du corps éviscéré de Rats, soit soumis à une réduction de l'apport énergétique de la ration (animaux restreints), soit normalement alimentés (animaux témoins), a été étudiée en mesurant l'évolution - de la teneur en acide désoxyribonucléique (ADN), proportionnelle au nombre de cellules, du rapport poids frais/ADN proportionnel à la taille des cellules - des rapports protéines/ADN et ARN/ADN.

En comparant témoins et restreints de même poids, il est montré que chez les animaux restreints :

- Le poids du foie est inférieur à celui des témoins, la concentration de l'ADN est peu modifiée ; concentration des protéines est légèrement augmentée, celle de l'ARN diminuée.

- La proportion des muscles est peu différente de ce qu'elle est chez les témoins ; mais les rôles respectifs de l'hyperplasie et de l'hypertrophie sont modifiés. Comme dans le foie, la concentration en protéines est augmentée, la concentration en ARN est diminuée.

- Les modifications constatées dans le corps éviscéré sont dues pour la plus grande part à la présence des muscles. La croissance de l'ensemble des autres tissus (osseux, épithéliaux, adipeux, etc.) est ralentie, mais son déroulement est peu modifié. Seule l'augmentation en protéines est un phénomène général.

\section{INTRODUCTION}

Un certain nombre de travaux ont été consacrés à l'influence des carences d'un ou plusieurs éléments essentiels de la ration, sur l'évolution de la teneur en acides désoxyribonucléique (ADN) et ribonucléique (ARN) des tissus animaux en croissance. Parmi ces éléments, les plus étudiés ont été les acides aminés (VANDERMEERS et al., I964-rg66 ; DuRAND et al., I966) et aussi les vitamines (TERROINE, I964).

De l'ensemble de ces travaux, il ressort que les carences entraînent un ralentissement de la synthèse des acides nucléiques. Cependant, la privation d'un élément 
nutritif indispensable entraîne généralement une diminution de l'appétit de l'animal et par conséquent, une sous-alimentation générale, dont l'action dépressive vient s'ajouter à celle de la carence elle-même. L'une des conséquences de la sous-alimentation est la diminution de l'apport énergétique de la ration.

Le but de ce travail est d'étudier les effets propres à cette diminution lorsque tous les autres facteurs alimentaires : protéines, minéraux et vitamines sont apportés en quantité largement suffisante. Il concerne 1'évolution de la teneur en acides nucléiques et en protéines $d u$ foie, des muscles et du corps éviscéré de rats dont la croissance est ralentie par la seule réduction des éléments énergétiques de la ration (lipides et glucides).

La variation des quantités d'ADN permet d'évaluer l'évolution du nombre de noyaux, et, par extension, de cellules, en appelant cellule l'ensemble d'un noyau et de la quantité moyenne de cytoplasme qu'il contrôle (DURAND et al., I965). Le rapport poids frais/ADN varie comme la taille moyenne des cellules. Les rapports protéines/ADN et ARN/ADN sont considérés comme deux critères de l'activité cellulaire.

\section{MATÉRIEI, ET MÉTHODES}

\section{I - Matériel animal}

Cinq lots de rats mâles (Wistar) sont utilisés. Ces animaux sont mis en expérience à $7 \circ \mathrm{g}$ (4 semaines). Un lot est abattu à ce poids. Deux lots reçoivent une alimentation permettant une croissance normale. Les animaux constituant ces lots sont dits " témoins ". Le premier de ces deux lots est sacrifié lorsque les rats pèsent $200 \mathrm{~g}$, le second, lorsque les animaux pèsent $400 \mathrm{~g}$. Deux lots reçoivent une alimentation diminuée en énergie ne permettant qu'une croissance ralentie. Les animaux constituant ces lots sont dits " restreints ". De même que précédemment, l'un des lots est sacrifié à $200 \mathrm{~g}$, l'autre à $400 \mathrm{~g}$.

Témoins et restreints peuvent ainsi être comparés à poids égal.

a) Consommation alimentaire et croissance.

TABLEAU I

Composition centésimale du régime " témoins "

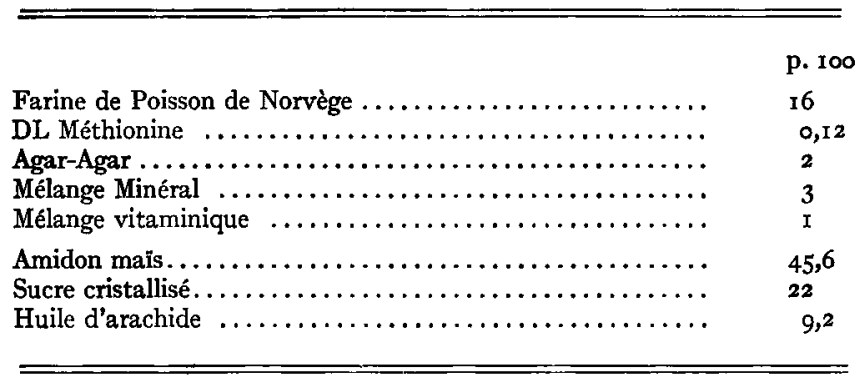

Les témoins reçoivent ad libitum un régime semi-synthétique contenant $13 \mathrm{p}$. Ioo de matières azotées; sa composition centésimale est donnée dans le tableau I. Les animaux sont pesés chaque matin et leur consommation est mesurée. La croissance obtenue est satisfaisante $: 6,5 \mathrm{~g}$ pendant la période ou la croissance est maximale. 
Les restreints sont astreints à suivre une courbe de croissance linéaire, correspondant à un gain de poids journalier de $2 \mathrm{~g}$; pour obtenir ce résultat, il est procédé de la manière suivante : les animaux reçoivent chaque jour la même quantité de protéines, de vitamines et de minéraux que consommeraient des animaux témoins de même poids : les besoins des restreints en ces éléments sont donc largement couverts. La quantité d'éléments énergétiques (amidon de maïs, sucre cristallisé et huile d'arachide) est ajustée chaque jour, de façon à ce que les animaux prennent seulement le nombre de grammes désiré. L'évolution des consommations des témoins et des restreints en fonction du poids des animaux est illustrée par les figures 2 et 3 . Il apparaît que pour assurer un gain de poids de $2 \mathrm{~g} /$ jour, la restriction énergétique doit être de moins en moins séveere dès que les animauxnt dépasse $200 \mathrm{~g}$.

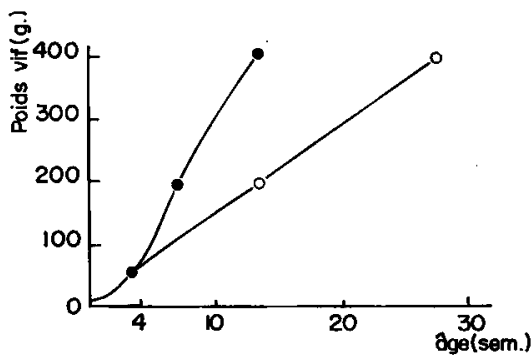

FIG. I. - Courbes de croissance des rals nourris d volonté (témoins $\bullet$ ) et des rats d croissance ralen(restreints o) par l'apport énergétique de la ration. L'apport énergétique a été ajusté chaque jour, de façon d ce que la courbe de croissance des restreints soit linéaire $(\Delta \mathrm{P}=2 \mathrm{~g} / \mathrm{jour})$.

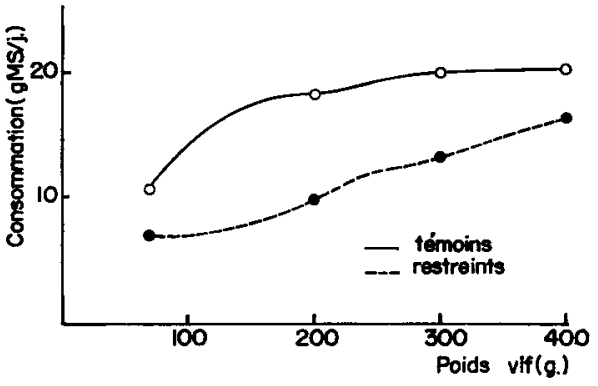

FIG. 2. - Quantités d'aliments ingérés par les rats nourris d volonté et par les rats dont le gain de poids quotidien est limité d $2 \mathrm{~g}$ par réduction de l'apport énergétique de la ration, en fonction de leur poids vif.

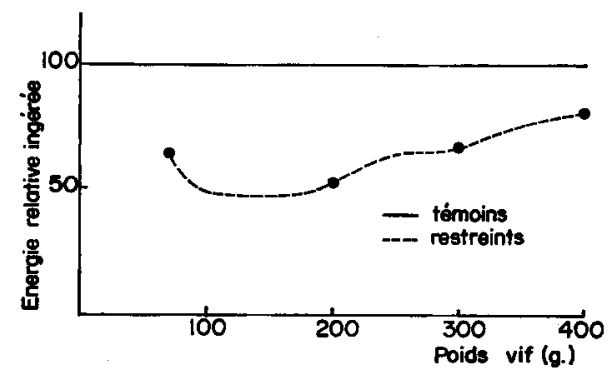

FIG. 3. - Quantité d'énergie de la ration nécessaire pour assurer aux rats restreints un gain de poids journalier de $2 \mathrm{~g}$, exprimée en $p .100$ de la quantité d'énergie consommée par les rats nourris d volonté.

b) Aspect des animaux. - Les animaux restreints présentent tous les signes extérieurs d'un excellent état physiologique et sont comparables aux animaux témoins de même poids.

c) Abattage et prélèvement. - Chaque animal est anesthésié à l'éther. Le foie est rapidement prélevé, rincé au sérum physiologique à o ${ }^{\circ} \mathrm{C}$, essuyé, pesé et plongé dans l'azote liquide. Tous les organes de l'abdomen et de la cage thoracique, ainsi que les organes génitaux sont enlevés. Il reste alors ce qu'on appelle l" "animal éviscéré ". Les muscles des membres postérieurs sont ensuite prélevés aussi rapidement que possible, l'ensemble de ces muscles d'une part et ce qu'il reste du corps éviscéré d'autre part sont pesés et refroidis par l'azote liquide.

Pour chaque lot, l'ensemble des foies, des muscles des membres postérieurs et des corps éviscérés sans ces muscles sont séparément rassemblés. On obtient ainsi trois échantillons qui seront analysés.

$$
\text { II - Méthodes analytiques }
$$

L'ensemble des méthodes analytiques conduisant au dosage de l'azote et des acides nucléiques des diverses parties prélevées, ont déjà été décrites (DuRAND et al., 1965). 


\section{RÉSULTATS}

\section{Expression des résultats.}

Les résultats exposés ci-dessous, concernant le corps éviscéré ont été calculés en utilisant les données correspondant à la musculature des membres postérieurs et celles correspondant au corps éviscéré sans cette musculature.

Les quantités d'acides nucléiques sont exprimées en micromoles $(\mu \mathrm{M})$ de leurs bases constitutives.

\section{Fore (tabl. 2).}

A poids d'animal égal, le foie d'un animal restreint est plus petit : de 29 p. Ioo quand l'animal pèse $200 \mathrm{~g}$, de $27 \mathrm{p}$. Ioo quand il pèse $400 \mathrm{~g}$. En revanche, sa concentration en protéines est plus élevée, comme en témoigne la valeur supérieure du rapport poids $\mathrm{SD}\left(^{1}\right) \mathrm{p}$. Ioo poids frais.

Lorsque le poids des animaux passe de 70 à $200 \mathrm{~g}$, la quantité d'ADN contenue est multipliée par 2,26 lorsque la croissance est normale, et seulement par $I, 59$ lorsque la croissance est ralentie. Lorsque le poids des animaux varie de 200 à $400 \mathrm{~g}$, la quantité d'ADN du foie est multipliée par I,45 dans le cas de la croissance normale, et par I,77 dans le cas de la croissance ralentie. Il en résulte que la quantité totale d'ADN

\section{TABLEAU 2}

Foie

Influence de l'apport énergétique de la ration sur les teneurs en acides nucléiques et en protéines

\begin{tabular}{|c|c|c|c|c|c|}
\hline & & Témoins & Restreints & Témoins & Restreints \\
\hline $\begin{array}{l}\text { Poids vif moyen }(\mathrm{g}) \ldots \ldots \\
\text { Age (semaines) } \ldots \ldots \ldots \\
\text { Nombre de rats } \ldots \ldots \ldots\end{array}$ & $\begin{array}{r}70 \\
4 \\
8\end{array}$ & $\begin{array}{r}200 \\
7 \\
8\end{array}$ & $\begin{array}{r}199 \\
13 \\
5\end{array}$ & $\begin{array}{c}404 \\
13,5 \\
9\end{array}$ & $\begin{array}{c}408 \\
27,5 \\
6\end{array}$ \\
\hline $\begin{array}{l}\text { Poids frais/rat (g) ....... } \\
\text { Poids frais \% poids vif ... } \\
\text { Poids SD (1) (g) ........ } \\
\text { Poids SD \% poids trais... }\end{array}$ & $\begin{array}{l}3,9 \\
6 \\
0,72 \\
18,5\end{array}$ & $\begin{array}{r}9,4 \\
4,7 \\
1,7 \\
18\end{array}$ & $\begin{array}{r}6,7 \\
3,4 \\
1,4 \\
21,3\end{array}$ & $\begin{array}{r}15,6 \\
3,9 \\
2,9 \\
19\end{array}$ & $\begin{array}{r}11,4 \\
2,8 \\
2,4 \\
20,9\end{array}$ \\
\hline $\begin{array}{l}\mathrm{ADN} / \mathrm{g} \text { SD ( } \mu \mathrm{M} \text {-bases) } \ldots \\
\mathrm{ADN} \text { total ( } \mu \mathrm{M} \text {-bases) } \ldots\end{array}$ & $\begin{array}{l}38,7 \\
27,9\end{array}$ & $\begin{array}{l}37,1 \\
63,1\end{array}$ & $\begin{array}{l}31,3 \\
43,8\end{array}$ & $\begin{array}{l}31,5 \\
91,3\end{array}$ & $\begin{array}{l}33 \\
79,2\end{array}$ \\
\hline $\begin{array}{l}\text { Poids frais (mg)/ADN }(\mu \mathrm{M}- \\
\text { bases) } \ldots \ldots \ldots \ldots \ldots \ldots \\
\text { Protéines (mg)/ADN ( } \mu \mathrm{M} \text { - } \\
\text { bases) } \ldots \ldots \ldots \ldots \ldots \ldots \\
\text { ARN/ADN } \ldots \ldots \ldots \ldots \ldots\end{array}$ & $\begin{array}{l}140 \\
23,9 \\
3,47\end{array}$ & $\begin{array}{l}149 \\
24,9 \\
3,70\end{array}$ & $\begin{array}{l}153 \\
29,6 \\
2,99\end{array}$ & $\begin{array}{l}171 \\
29,4 \\
3,20\end{array}$ & $\begin{array}{c}144 \\
28,0 \\
2,99\end{array}$ \\
\hline
\end{tabular}

(1) $\mathrm{SD}=$ Poids sec et dégraissé. 
contenue dans les foies des animaux restreints est diminuée de $30 \mathrm{p}$. Ioo quand les animaux pèsent $200 \mathrm{~g}$, et de I4 $\mathrm{p}$. Ioo seulement quand ils pèsent $400 \mathrm{~g}$. L'écart creusé au cours des premières semaines qui suivent la mise au régime restreint tend donc à se combler peu à peu.

Le rapport poids frais/ADN ne varie guère, quels que soient le stade et le type de croissance : lorsque les animaux pèsent $200 \mathrm{~g}$ il est identique chez les restreints et les témoins, lorsque les animaux pèsent $400 \mathrm{~g}$, il est plus petit d'environ $\mathrm{I} 5 \mathrm{p}$. Ioo chez les restreints. Autrement dit, les quantités d'ADN par unité de poids frais sont à peu près égales dans les foies des restreints et des témoins.

Le rapport protéines/ADN est plus élevé (I5 p. IOo) dans le foie des animaux restreints, contrairement au rapport $\mathrm{ARN} / \mathrm{ADN}$, toujours inférieur.

\section{MUSCLES DES MEMBRES POSTÉRIEURS (tabl. 3)}

Quand le poids des animaux passe de 70 à $200 \mathrm{~g}$, le poids des muscles des témoins est multiplié par 3,66, celui des restreints par 4 .

Quand le poids des animaux passe de 200 à $400 \mathrm{~g}$, le poids des muscles des témoins est multiplié par 2 environ, celui des restreints par $\mathrm{r}, 7$ seulement. Ainsi, la légère avance $(8,5 \mathrm{p}$. IOo) prise par les animaux restreints au cours des semaines qui suivent la mise au régime a pratiquement disparu quand les animaux atteignent $400 \mathrm{~g}$. Le rapport poids SD p. Ioo poids frais est supérieur de Io à I2 $\mathrm{p}$. I00 chez les animaux restreints, ce qui indique une augmentation du même ordre de la concentration en protéines.

Entre 70 et $200 \mathrm{~g}$ la quantité totale d'ADN contenue dans les muscles est multipliée par 2,4 chez les témoins et par I,9 chez les restreints. Entre 200 et $400 \mathrm{~g}$ par

\section{TABLEAU 3}

Musculature des membres postérieurs du rat

Influence de l'apport énergétique de la ration sur les teneurs en acides nucléiques et en protéines

\begin{tabular}{|c|c|c|c|c|c|}
\hline & & Témoins & Restreints & Têmoins & Restreints \\
\hline $\begin{array}{l}\text { Poids vif (g) } \ldots \ldots \ldots \ldots \\
\text { Age (semaines) } \ldots \ldots \ldots \\
\text { Nombre de rats } \ldots \ldots \ldots\end{array}$ & $\begin{array}{r}70 \\
4 \\
8\end{array}$ & $\begin{array}{r}200 \\
7 \\
8\end{array}$ & $\begin{array}{r}199 \\
13 \\
5\end{array}$ & $\begin{array}{c}404 \\
13,5 \\
9\end{array}$ & $\begin{array}{c}408 \\
27,5 \\
6\end{array}$ \\
\hline $\begin{array}{l}\text { Poids frais } / \text { rat }(g) \ldots \ldots . \\
\text { Poids frais } \% \text { poids vif } \\
\text { Poids SD }(\mathbf{1})(g) \ldots \ldots \ldots \\
\text { Poids SD } \% \text { poids frais. . }\end{array}$ & $\begin{array}{r}4,7 \\
6,6 \\
0,8 \\
17,7\end{array}$ & $\begin{array}{r}17,2 \\
8,6 \\
3,3 \\
19,2\end{array}$ & $\begin{array}{r}18,8 \\
9,5 \\
4,1 \\
21,9\end{array}$ & $\begin{array}{r}34 \\
8,4 \\
7,2 \\
21,2\end{array}$ & $\begin{array}{r}32,5 \\
8,1 \\
7,6 \\
23,4\end{array}$ \\
\hline $\begin{array}{l}\mathrm{ADN} / \mathrm{g} \text { SD }(\mu \mathrm{M} \text {-bases }) \ldots \\
\mathrm{ADN} \text { total }(\mu \mathrm{M} \text {-bases }) \ldots\end{array}$ & $\begin{array}{l}19,3 \\
15,4\end{array}$ & $\begin{array}{l}11,3 \\
37,3\end{array}$ & $\begin{array}{r}7,2 \\
29,8\end{array}$ & $\begin{array}{r}6,7 \\
48,2\end{array}$ & $\begin{array}{r}6,0 \\
46,0\end{array}$ \\
\hline $\begin{array}{l}\text { Poids frais }(\mathrm{mg}) / \mathrm{ADN}(\mu \mathrm{M}- \\
\text { bases) } \ldots \ldots \ldots \ldots \ldots \ldots \\
\text { Protéines }(\mathrm{mg}) / \mathrm{ADN}(\mu \mathrm{M} \text { - } \\
\text { bases) } \ldots \ldots \ldots \ldots \ldots \ldots \\
\text { ARN/ADN } \ldots \ldots \ldots \ldots \ldots\end{array}$ & $\begin{array}{l}305 \\
48 \\
1,76\end{array}$ & $\begin{array}{l}461 \\
82 \\
2,04\end{array}$ & $\begin{array}{l}630 \\
128 \\
1,76\end{array}$ & $\begin{array}{l}705 \\
138 \\
2,26\end{array}$ & $\begin{array}{l}709 \\
154 \\
1,83\end{array}$ \\
\hline
\end{tabular}

(I) $\mathrm{SD}=$ Poids sec et dégraissé. 
I,3 chez les témoins et par I,5 chez les restreints. Ainsi à $200 \mathrm{~g}$, la quantité d'ADN contenue dans les muscles des restreints est inférieure de $20 \mathrm{p}$. Ioo à celle contenue chez les témoins. Plus tard, l'écart s'atténue, à tel point qu'il n'est plus appréciable (inférieur à 4 p. roo) lorsque les animaux atteignent $400 \mathrm{~g}$. Le rapport poids frais/ADN chez les restreints de $200 \mathrm{~g}$ est supérieur de $27 \mathrm{p}$. roo à ce qu'il est chez les témoins de même poids. Lorsque les animaux pèsent $400 \mathrm{~g}$, l'écart a complètement disparu et les rapports sont équivalents.

Le rapport protéines/ADN est plus élevé chez les animaux restreints. Cette supériorité est très nette à $200 \mathrm{~g}$, puisqu'elle atteint $36 \mathrm{p}$. Ioo ; elle s'atténue par la suite, et n'est plus que de Io p. IO0 à $400 \mathrm{~g}$. Le rapport ARN/ADN est plus faible chez les restreints, où sa valeur ne varie pratiquement pas au cours de la période étudiée. Chez les témoins, en revanche, elle augmente notablement.

\section{CoRPS ÉviscérÉ (tabl, 4).}

Il ressort de l'examen du tableau 4 que les corps éviscérés des animaux à croissance ralentie par réduction de l'apport énergétique de la ration sont plus riches en

TABLEAU 4

Corps éviscéré du rat

Comparaison entre des animaux à croissance normale et à croissance ralentie uniquement par réduction de l'apport énergétique de la ration

\begin{tabular}{|c|c|c|c|c|c|}
\hline$\ddots$ & & Témoins & Restreints & Témoins & Restreints \\
\hline $\begin{array}{l}\text { Poids vif }(g) \ldots \ldots \ldots \ldots \\
\text { Age (semaines) } \ldots \ldots \ldots \ldots \\
\text { Nombre de rats } \ldots \ldots \ldots\end{array}$ & $\begin{array}{r}70 \\
4 \\
8\end{array}$ & $\begin{array}{r}200 \\
7 \\
8\end{array}$ & $\begin{array}{r}199 \\
13 \\
5\end{array}$ & $\begin{array}{c}404 \\
13,5 \\
9\end{array}$ & $\begin{array}{c}408 \\
27,5 \\
6\end{array}$ \\
\hline
\end{tabular}

a) Composition globale



b) Teneurs en acides nucléiques et en protéines

\begin{tabular}{|c|c|c|c|c|c|}
\hline Poids frais/rat $(g) \ldots \ldots$ & 52,9 & 162 & 166 & 349 & 349 \\
\hline Poids frais $\%$ poids vif. .. & 75,4 & 81 & 83,5 & 86,4 & 85,5 \\
\hline Poids SD $\left({ }^{1}\right)(g) \ldots \ldots \ldots$ & 9,6 & 29,6 & 37,0 & 62,8 & 70,9 \\
\hline Poids SD $\%$ poids frais... & 18,1 & 18,3 & 22,3 & 18,0 & 20,3 \\
\hline ADN/g SD ( $\mu \mathrm{M}$-bases) . . . & 34,5 & 20,4 & 14,8 & 13,2 & 11,5 \\
\hline ADN total ( $\mu$ M-bases). . . . & 331 & 604 & 548 & 829 & 812 \\
\hline $\begin{array}{c}\text { Poids frais }(\mathrm{mg}) / \mathrm{ADN}(\mu \mathrm{M}- \\
\text { bases }) \ldots \ldots \ldots \ldots \ldots \ldots\end{array}$ & 158 & 268 & 303 & 421 & 430 \\
\hline Protéines mg)/ADN ( $\mu \mathrm{M}$ - & & & & & \\
\hline bases) $\ldots \ldots \ldots \ldots \ldots$ & 26,8 & 45,3 & 62,5 & 70,1 & 80,4 \\
\hline ARN/ADN $\ldots \ldots \ldots \ldots$ & 1,19 & 1,30 & 1,19 & 1,18 & 1,19 \\
\hline
\end{tabular}

(1) $\mathrm{SD}=$ Poids sec et dégraissé. 
protéines et en minéraux que les animaux témoins de même poids. Par rapport aux témoins, la teneur en minéraux est multipliée par $I, 2$ à $I, 3$ et la teneur en protéines par I,I. Ces augmentations assez importantes en valeur relative, mais faibles dans l'absolu, n'ont que peu de répercussions sur les teneurs en eau et en lipides.

Le poids frais des corps éviscérés est approximativement égal chez les témoins et les restreints ; le poids SD est plus élevé chez ces derniers, tant à $200 \mathrm{~g}$ qu'à $400 \mathrm{~g}$.

Entre 70 et $200 \mathrm{~g}$, la quantité d'ADN est multipliée par I,82 chez les témoins et par I,65 chez les restreints et, entre 200 et $400 \mathrm{~g}$, par I,37 chez les témoins et $\mathrm{I}, 48$ chez les restreints. Ainsi à $200 \mathrm{~g}$, la quantité totale d'ADN est supérieure de $9 \mathrm{p}$. roo chez les restreints, mais cette différence a pratiquement disparu (à 2 p. roo près) quand les animaux atteignent $400 \mathrm{~g}$. Les animaux de même poids ayant des corps éviscérés approximativement de même poids, il en résulte évidemment que le rapport poids frais/ADN varie comme l'inverse de l'ADN : il est plus élevé chez les animaux restreints de $200 \mathrm{~g}$ que chez les témoins correspondants.

Le rapport protéines/ADN est plus élevé chez les restreints : de 27 p. I00 à 200 et $\mathrm{I} 3 \mathrm{p}$. Ioo à $400 \mathrm{~g}$. Le rapport ARN/ADN est peu modifié par la restriction énergétique et reste, dans l'un et l'autre cas, voisin de $I, 2$.

\section{DISCUSSION}

Foie.

Lorsqu'on ralentit la croissance d'un animal uniquement par réduction de l'apport énergétique de la ration, les besoins en protéines, vitamines et minéraux étant par ailleurs largement couverts, le poids du foie des animaux restreints est inférieur de 25 à 30 p. roo à celui des animaux normaux de même poids vif. En revanche, la restriction énergétique entraîne une augmentation notable de la concentration en protéines de 1'organe.

Dans une précédente publication (DuRAND et al., 1965) il a été montré que, dans les conditions d'une alimentation satisfaisante et compte tenu des variations du taux de polyploidie des noyaux, la croissance du foie est due principalement à l'hyperplasie, le rôle de l'hypertrophie n'étant cependant pas négligeable surtout à partir du moment où l'animal atteint 1'âge, ou plutôt le poids"de la maturité sexuelle. Dans le cas d'un ralentissement de la croissance par réduction de l'apport énergétique de la ration, les variations du taux de polyploidie ne sont pas connues. Il n'est donc pas possible d'évaluer les rôles respectifs de l'hyperplasie et de l'hypertrophie aux divers stades de la croissance. Quoi qu'il en soit, la synthèse de l'ADN est ralentie chez les restreints à un moment où elle est très rapide chez les animaux normaux : mais le manque d'intensité est compensé par la durée, si bien que le retard accumulé est peu à peu comblé et il est probable qu'il disparaît complètement lorsque les animaux atteignent leur poids maximal : les routes empruntées sont différentes, mais le point d'arrivée est le même. Ce phénomène de ralentissement de synthèse est encore accentué en ce qui concerne l'ARN puisque le rapport ARN/ADN est diminué. Sa valeur est inférieure ou égale à 3 , alors qu'elle est toujours égale ou supérieure à 3,2 dans le cas d'une croissance rapide. En revanche, il est remarquable qu'à poids 
égal d'animal, alors que la quantité totale d'ADN est diminuée de $27 \mathrm{p}$. Ioo et la quantité totale d'ARN de 30 à $40 \mathrm{p}$. Ioo, la quantité de protéines de l'organe n'est diminuée que de $I 8 \mathrm{p}$. Ioo : il apparaît donc que la carence énergétique ralentit davantage la synthèse des acides nucléiques que celle des protéines.

Muscles.

La croissance des muscles est un peu moins ralentie par la restriction énergétique que celle de 1'animal entier. La teneur en protéines est notablement augmentée.

Aucune trace de polyploïdie n'ayant été détectée dans le muscle (LASH et al., I957; ENEsco et Della PUDDy, I964), on peut considérer que la quantitéd'ADN varie comme le nombre, et le rapport poids frais/ADN comme la taille des cellules.

L'analyse, suivant le mode de calcul exposé en détail dans une précédente publication (Durand et al., I965), des rôles respectifs de 1'hyperplasie et de l'hypertrophie dans le cas de la croissance musculaire normale d'une part, et ralentie d'autre part, est rapportée dans le tableau 5 .

Il ressort de ces résultats que la faible croissance permise par le régime restreint est assurée dans un premier temps principalement par l'hypertrophie qui paraît donc être prioritaire sur 1'hyperplasie. Dans les conditions auxquelles ont été soumis les animaux restreints, l'hypertrophie a pu évoluer d'une façon presque comparable chez les restreints et les témoins : on remarque en effet que les muscles des restreints de $200 \mathrm{~g}$ et des témoins de $400 \mathrm{~g}$, dont 1'âge est approximativement le même, ont un rapport poids frais/ADN peu différent (630 au lieu de 705, soit io p. Ioo alors que la quantité d'ADN diffère de 37 p. IOO).

TABLEAU 5

Croissance musculaire

Influence de l'apport énergétique de la ration sur les rôles respectifs de l'hyperplasie et de l'hypertrophie

\begin{tabular}{|c|c|c|c|c|}
\hline Régime & \multicolumn{2}{|c|}{ Témoin } & \multicolumn{2}{|c|}{ Restreint } \\
\hline Poids vif ...... & de 70 à $200 \mathrm{~g}$ & de 200 à $400 \mathrm{~g}$ & de 70 à $200 \mathrm{~g}$ & de 200 à $400 \mathrm{~g}$ \\
\hline $\begin{array}{c}\text { Intervention } \\
\text { plasie }(\%)\end{array}$ & 67 & 37 & 48 & 78 \\
\hline $\begin{array}{l}\text { Intervention de l'hyper- } \\
\quad \text { trophie }(\%) . \ldots \ldots \ldots \ldots\end{array}$ & 33 & 63 & 52 & 22 \\
\hline
\end{tabular}

Cependant, chez les restreints, au-delà de $200 \mathrm{~g}$, tout se passe comme si la taille moyenne des cellules approchant le maximum, l'hypertrophie ne pouvait plus assurer la croissance que pour une part très faible. Dès lors, l'hyperplasie, loin d'être arrivée à son terme et qui se poursuit à un rythme lent mais continu, assure la continuation de la croissance. 
Finalement, chez les animaux de $400 \mathrm{~g}$, lorsque la croissance musculaire touche presque à sa fin, le nombre et la taille des cellules sont identiques chez les témoins et les restreints.

Comme dans le cas du foie, l'augmentation de la quantité d'ARN est plus inhibée que celle de l'ADN, comme en témoigne la faible valeur du rapport $A R N / A D N$. II serait intéressant de savoir si tous les ARN sont concernés de la même façon.

Corps éviscéré.

a) Composition globale. - A poids d'animal égal, le corps éviscéré des restreints, est tojujours plus riche en protéines et surtout en minéraux. L'engraissement des animaux est normal et la teneur en eau légèrement diminuée. Mais, dans 1'ensemble la composition globale n'est pas considérablement modifiée.

b) Hyperplasie et hypertrophie. - Entre 70 et $200 \mathrm{~g}$, la quantité d'ADN, donc de cellules, est légèrement diminuée chez les restreints (9 p. IOo). Or, le corps éviscéré est constitué, pour une large part, de muscles dont on a vu que chez les animaux restreints de $200 \mathrm{~g}$, le déficit en $\mathrm{ADN}$ était de $20 \mathrm{p}$. Ioo. Il semble donc, en première analyse que le déficit en $\mathrm{ADN}$ de la musculature, peut expliquer à lui seul une grande part du déficit du corps éviscéré entier.

Les données rassemblées dans le tableau 6 , obtenues à partir de nos résultats et de ceux de CHEEK et al. (I965), montrent que l'augmentation de la quantité $\mathrm{d}^{\prime} \mathrm{ADN}$ est fortement réduite dans la musculature des restreints (33 p. IOO).

\section{TABLEAU 6}

Evolution de l'ADN dans la musculature et dans l'ensemble des autres tissus (corps éviscéré sans musculature) chez le Rat soumis à une réduction de l'apport énergétique de la ration, au cours des premiers stades de la croissance

\begin{tabular}{|c|c|c|c|}
\hline & & 'l'émoins & Restreints \\
\hline 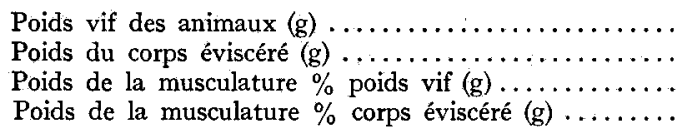 & $\begin{array}{l}70 \\
52,9 \\
30 \\
40\end{array}$ & $\begin{array}{r}200 \\
162 \\
38 \\
47\end{array}$ & $\begin{array}{l}200 \\
166 \\
41,5 \\
50\end{array}$ \\
\hline $\begin{array}{l}\text { Poids de la musculature }(\mathrm{g}) \ldots \ldots \ldots \ldots \ldots \ldots \ldots \ldots \ldots \ldots \ldots \ldots \ldots \ldots \ldots \ldots \ldots \ldots \ldots \ldots \ldots \ldots \ldots \ldots \ldots \ldots \ldots \\
\text { ADN de la musculature }(\mu \mathrm{M}) \ldots \ldots \ldots \ldots \ldots \ldots \ldots \ldots\end{array}$ & $\begin{array}{r}21 \\
69 \\
304\end{array}$ & $\begin{array}{r}76 \\
165 \\
458\end{array}$ & $\begin{array}{r}83 \\
132 \\
630\end{array}$ \\
\hline $\begin{array}{l}\text { Poids du corps éviscéré sans musculature }(g) \ldots \ldots \ldots \ldots \\
\mathrm{ADN} d u \text { corps éviscéré sans musculature }(\mu \mathrm{M}) \ldots \ldots \ldots \\
\text { Poids du corps éviscéré sans musculature }(\mathrm{g}) / \mathrm{ADN}(\mu \mathrm{M})\end{array}$ & $\begin{array}{r}32 \\
262 \\
122\end{array}$ & $\begin{array}{r}86 \\
439 \\
196\end{array}$ & $\begin{array}{r}83 \\
416 \\
200\end{array}$ \\
\hline
\end{tabular}

Dans le corps éviscéré sans musculature, 1'augmentation de la quantité d'ADN est réduite de $\mathrm{I} 4 \mathrm{p}$. Ioo; mais en même temps, l'augmentation du poids du corps éviscéré sans musculature est également légèrement diminuée chez les restreints, si bien que contrairement à ce qui se produit dans les muscles, la taille des cellules n'est pratiquement pas changée entre témoins et restreints. 
On en déduit que la restriction énergétique agit différemment sur le tissu musculaire et l'ensemble des autres tissus : alors que la croissance de ces derniers est seulement ralentie, celle de la musculature est en même temps modifiée par une sorte de renversement de l'équilibre hyperplasie-hypertrophie.

La comparaison des rapports ARN/ADN des muscles d'une part et de la carcasse d'autre part faisait déjà pressentir que le tissu musculaire présentait des particularités : en effet, le rapport ARN/ADN n'est pas modifié dans les tissus autres que le musculaire.

\section{CONCLUSION}

Sauf en ce qui concerne le foie, dont la taille est sans doute liée à la quantité de nutriments qu'il doit métaboliser, on remarque que lorsque les animaux ont atteint le poids de $400 \mathrm{~g}$, témoins et restreints se retrouvent au même point quant au nombre et à la taille des cellules de leurs tissus.

En fait, la restriction de l'apport énergétique de la ration telle qu'elle a été pratiquée ici n'a fait que retarder l'arrivée à 1'âge adulte, si l'on définit celui-ci comme étant 1'état de 1'animal qui a acquis la plus grande part du capital cellulaire correspondant à ses capacités génétiques. L'animal restreint peut en effet augmenter le nombre de ses cellules à un âge où l'animal à croissance rapide a perdu en grande partie ce pouvoir (au-delà de 13 semaines).

Tout se passe comme si les animaux restreints demeuraient plus longtemps à un stade juvénile. Ceci est d'ailleurs confirmé par leur aspect extérieur et leur composition corporelle.

I a question se pose de savoir pendant combien de temps ce pouvoir de multiplication cellulaire peut être conservé, et s'il peut être conservé dans sa totalité.

Rę̧u pour publication en mars 1967.

\section{SUMMARY}

GROWTH OF TISSUES OF RATS AND REDUCTION OF ENERGY SUPPLIED BY THE RATION ; INFLUENCE ON NUCLEIC ACID CONTENT

Growth of liver, muscles of the hind limbs and the eviscerated carcase of rats given a reduced supply of energy in their ration (restricted animals) and of rats fed normally (control animals) was studied by measuring : - the changes in the content of deoxyribonucleic acid (DNA), proportional to the number of cells - the ratio of fresh weight to DNA, proportional to the size of the cells - the ratios of protein to DNA and of ribonucleic acid (RNA) to DNA.

The animals weighed about $70 \mathrm{~g}$ and were 4 weeks old at the start of the experiment. Controls were fed to appetite on a balanced diet with 13 per cent crude protein ( $N$ X 6. 25). The restricted rats got the same amounts of protein, minerals and vitamins daily as controls of the same weight would eat, but by reducing the supply of carbohydrates and lipids animals were made to follow a linear growth curve corresponding to a daily gain of only $2 \mathrm{~g}$. weights.

Groups of control and restricted rats were killed at 200 or at $400 \mathrm{~g}$ and compared at equal

Liver (table 2). - At $200 \mathrm{~g}$, the divers of restricted rats weighed 29 per cent less than those of controls. The amount of DNA per unit fresh weight was similar in both groups. In the restricted rats 
the concentration of protein increased and that of RNA decreased. From 200 to $400 \mathrm{~g}$ the wide differences seen during the first weeks after the start of the trial tended to become less.

Muscles of the hind limbs (tables 3 and 5). - At $200 \mathrm{~g}$ the weight of muscle differed little between control and restricted rats. The amount of DNA per unit fresh weight was considerably less ( 27 per cent). Between 70 and $200 \mathrm{~g}$, the respective rates of hyperplasia and hypertrophy were changed by restriction of energy which, reducing particularly the rate of the former, increased the relative importance of the latter. In the restricted rats the concentration of protein increased and that of RNA diminished. Between 200 and $400 \mathrm{~g}$, the difference between control and restricted rats became less ; the restricted rats retained the ability to synthesize DNA in large amounts to a late age (after I3 weeks).

Eviscerated carcase (tables 4 and 6). - At $200 \mathrm{~g}$ the amount of DNA was slightly reduced (9 per cent) in restricted rats ; concentration of protein was increased and that of RNA was reduced. Analysis of the results showed that muscular tissue, which was 40 to 50 per cent of the eviscerated car. case, was responsible for most of the change, but the high protein content was a characteristic common to all the tissues of the restricted rats. Between 200 and $400 \mathrm{~g}$ the differences became less and at $400 \mathrm{~g}$ it became difficult to distinguish between restricted and control rats, although the former were twice as old as the latter.

It was concluded that slowing down of growth rate by restriction of energy in the ration retards the age at which the adult stage is reached. The restricted animal can in effect increase the number of its cells at an age where the fast growing animal, which had already formed the total number of cells corresponding to its genetic capacity, no longer has that ability.

\section{RÉFÉRENCES BIBLIOGRAPHIQUES}

Cheek D. B., Powell G. K., ScotT R. E., 1965. Growth of muscle mass and skeletal collagen in the Rat. I. Normal growth. II. The effect of ablation of pituitary thyroid or testes. Bull. Hopkins Hosp., 116, 378-395.

Durand G., Fauconneau G., Penot E., I965. Étude biochimique de la croissance de l'intestin grêle, du foie et de la carcasse du Rat ; rôles respectifs de la multiplication et du grandissement cellulaires. Ann. Biol. anim. Biochim., Biophys., 5, 163-187.

Durand G., Fauconneau G., Penot E. 1966. Croissance des tissus du Rat et qualité des protéines alimentaires, influence sur le nombre et la taille des cellules. Ann. Biol. anim. Biochim. Biophys., 6, 389-409.

ENEsco M., PUDDY D., 1964. Increase in the number of nuclei and weight in skeletal muscle of Rats of various ages. Am. J. Anat., 114, 235-244.

Lash J. W., Holtzer H., Swift H., 1957. Regeneration of mature skeletal muscle. Anat. Rec., 128, $679-693$.

Terroine $T$., 1964. Le contrôle vitaminique de la irichesse en acides nucléiques tissulaires. I. Biotine. Arch. Sci. Physiol., 18, 363-382.

Vandermeers A., Christophe J., I964. Influence d'une carence en lysine sur le pancréas du Rat. Arch. internation. Physiol. Biochim., 72, 701-703.

Vandermeers-Piret M. C., Christophe J., ig64. Retentissement d'une carence en lysine sur le foie du Rat. Arch. internation. Physiol. Biochim., "72, 703-705.

Vandermeers-Piret M. C., Pokorni E., Wodon C., Christophe J., 1966. Acides nucléiques et protéines du foie de Rat carencé en lysine et en thréonine ou récupérant de cette carence. Bull. Soc. Chim. Biol., 48, 525-546. 